\title{
Real-Time Recognition of Handwritten Digits in FPGA Based on Neural Network with Fixed Point Calculations
}

\author{
R.A. Solovyev, D.V. Telpukhov, A.G. Kustov, V.S. Rukhlov, T.Y. Isaeva \\ Institute for Design Problems in Microelectronics of RAS, Moscow, turbo@ippm.ru
}

\begin{abstract}
In this paper convolutional neural networks for video stream processing using hardware with limited computing resource are studied. To solve problems of hardware performance, we propose to move from software computing to hardware implementation, and also move from fixed-point to floating point calculations. A set of methods for neural network design was proposed aimed at maximal network performance and accuracy in case of fixed point calculations. As the example, we describe solving of a specific practical task. We show how existing datasets can be adapted for another task with different data. An example of project development for prototyping in FPGA using camera and output device is given. Final project implemented with the use of the proposed methods successfully works in real time on De0Nano debug card based on Cyclone IV FPGA.
\end{abstract}

Keywords - convolutional neural networks (CNN), FPGA, fixed point calculations, 2D convolution.

\section{INTRODUCTION}

Recent research in the field of neural networks shows that they meet the challenge of classification and processing of images, audio and video data, and in some cases even do better than humans [1, 2]. Most modern architectures incorporate convolution blocks (e.g., VGG [1], Inception [3], ResNet [4], U-Net [5]). Such networks are called convolutional neural networks (CNN). Note that task dimension and computational complexity while classification is so great that even powerful generalpurpose CPUs cannot cope with this amount of computations. So, powerful and thus expensive GPUs (graphic processors) are used for proper work with modern neural networks [6]. This is especially true for real time video processing.

Properties of some structures of neural networks with very high accuracy of image classification are easily transferred to hardware platform:

1) High regularity - all layers have similar structure (Conv3x3, Conv1x1, MaxPooling, Fully-Connected, GlobalAvgPooling).

2) Small dimension of convolution block: 3x3.

3) Relu activation (comparing value with 0), in contrast to previously popular activation functions Sigmoid and Tanh, which have greater computational complexity.

4) Due to high regularity, the size of neural network can be flexibly adjusted; for example, due to different number of convolution blocks (the more there are, the faster the neural network operates). For example, in case of FPGA or VLSI, this allows us to flash the same neural network into different crystals, with different operation rate.

There is also such direction as design of neural networks for mobile devices: MobileNets [7], SqueezeNet [8]. Their characteristic features are small number of weights and relatively small number of arithmetic operations. However, they also perform at software level and use floating-point calculations. For devices that require high performance such as devices for real time video processing (at speed of 30 frames per second), even the use of mobile networks may be insufficient, if only significant optimization is not applied.

To use neural networks at the stage when we already have a trained model in a real device, we can apply a set of optimization operations; this can speed up calculations several times. There are already a number of techniques for this, for example, compression of weights [9] or calculations on low-bit data [10].

Since the demand for equipment for neural networks processing is constantly growing, development of special hardware units for use in VLSI and FPGAs is required to speed up calculations. Calculations speed up can be achieved due to the following features:

1) Hardware unit for convolution calculation works faster than convolution performed at software level.

2) Move from floating point to fixed point.

3) Decrease of computations dimensionality while keeping acceptable accuracy.

4) Reduction of part of neural network preserving classification accuracy.

5) Modifications of neural network structure with a slight decrease in accuracy (or even without it) with increase of performance and decrease in size of hardware implementation and stored weights.

6) Use of multiple convolutional blocks working in parallel.

\section{PREPARING OF TRAINING DATASET}

There is familiar MNIST data set [12] for the problem of digit recognition. However, it is very different from camera images (Fig. 1). For this reason, changes to MNIST are required. 

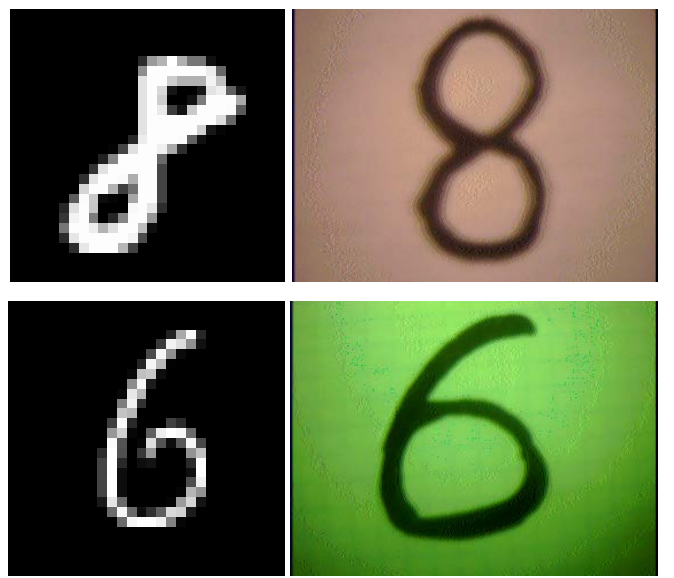

Fig. 1. Images from MNIST set (left) and images from camera (right)

Major differences:

1) MNIST images are light digits over dark background rather than dark digits over light background for camera image.

2) Camera image comes in color.

3) Size of MNIST image is $28 \times 28$ pixels, while camera image size is 320x240 pixels.

4) There are noise and artifacts in camera image, digits can be shifted and slightly rotated. As to digits from MNIST dataset, they are always placed strictly in the center and background is homogeneous and perfectly black.

5) As opposed to MNIST, we also have to add a separate eleventh class: "image without digits".

Maximum recognition accuracy for MNIST set is extremely high - modern networks recognize numbers with accuracy that exceeds $99.5 \%$ [13]. Thus we decided to reduce camera images to $28 \times 28$ pixels and convert images to grayscale. By this, the following problems are solved:

1) There is no significant loss in accuracy, as even for small images figures are still easily recognized by humans.

2) Color does not matter for uniform figures; it does not affect their recognition.

3) Noisy image from camera becomes clearer when neighboring pixels are reduced and averaged. Some of the noise is neutralized.

As image transformation is performed at hardware level, we have to think in advance of minimum set of arithmetic functions that effectively convert image to the desired form.

This is the algorithm for such conversion:

1) We take only central part of $224 \times 224$ pixels from $320 \times 240$ pixels of initial image. Number 224 is chosen because $224=28 * 8$, which subsequently allows easy transition to the desired image size.

2) Then the selected image part is converted to grayscale. Because of the peculiarities of human visual perception
[14], we take weighted average with coefficients about $60 \%$ for green, $30 \%$ for red and $10 \%$ for blue rather than simple average. To facilitate conversion at hardware level, we use the following formula:

$$
\mathrm{BW}=(8 * \mathrm{G}+5 * \mathrm{R}+3 * \mathrm{~B}) / 16
$$

Multiplication by 8 and division by 16 (which is even more important) are implemented using simple shifting.

3) Further, $224 \times 224$ image is split into $8 \times 8$ blocks. We calculate average value for each of these blocks and form corresponding pixels in 28x28 image.

This algorithm works very fast at hardware level.

Method of generating augmented images on the fly is applied so that we could use images from MNIST database for neural network training. This method implies that when creating next mini-batch for training, a set of various filters is applied to each image. This technique is used to increase dataset size with little effort, as well as to bring dataset to the required form, as in our case.

The following set of filters was used:

1) Color inversion.

2) Random rotation by 10 degrees in both directions.

3) Random increase or reduction of image by 4 pixels.

4) Random variation of image intensity (from 0 to 80 ).

5) Random noise insertion (from 0 to 10 percent).

Optionally, real images obtained from camera were added into training mini-batches.

\section{DESIGN OF NEURAL NETWORK WITH IMPROVED PERFORMANCE}

Typical structure of convolutional neural network is shown in Fig. 2. Later, some improved structures involving branching appeared [3-4]; however, the essence remained the same: image size decreases from layer to layer and number of filters grows. A set of characteristics is formed at the end of convolutional network, which are fed to classification layer (or layers) and output neurons report the probability that image belongs to a specific class.

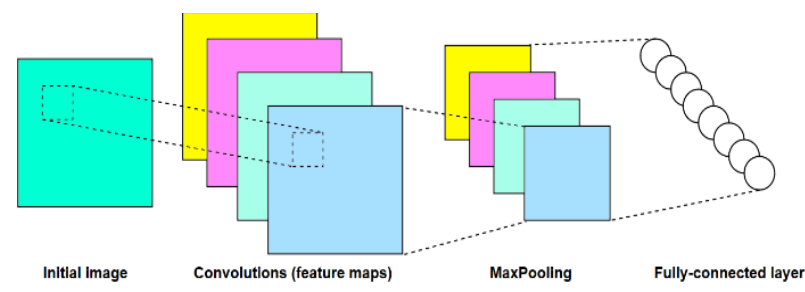

Fig. 2. Structure of convolution neural network

We propose the following set of rules for neural network design aimed at minimization of total number of stored weights (which is critical for mobile systems) and facilitating transfer to fixed-point calculations.

1) Minimize number of fully connected layers, which consume major part of memory for storing weights. Ideally, there should be one such layer — the last one. 
2) If possible, reduce number of filters of each convolution layer. Since each such reduction can degrade network properties, it is important to find good tradeoff between accuracy and number of weights.

3) Stop using bias. It is important when passing from floating point to fixed point, because adding a constant hinders monitoring value range; moreover, bias rounding error over each layer tends to accumulate.

4) Use simple type activation. The most suitable activation type is RELU. We should avoid such activation types as sigmoid, tahn, etc. that imply division and exponentiation and other functions that are difficult for hardware implementation.

5) Minimize number of heterogeneous layers so that one hardware unit could perform calculations at large number of flow stages.

Before translating neural network to hardware, we should train it on a prepared data set and build its mathematical model for testing. We created mathematical model based on Python and module for processing Keras neural networks with Tensorflow backend. In our previous publication [15] we proposed network architecture VGG Simple based on the same principles as effective neural network VGG16.

With its high performance, major challenge of this model is the number of weights, which exceeds FPGA capacity; also, exchange with external memory does not reach the desired speed. Additionally, this model contains bias, which also needs to be stored, and which requires extra processing blocks and tends to accumulate error in passing to fixed point representation. Therefore, we modified this structure, namely, eliminated large fully connected layers and bias. Also we added GlobalMaxPooling layer instead of GlobalAvgPooling layer, which is traditionally used in neural networks such as ResNet50. Efficiency of these layers is approximately the same, and, on computational side, finding maximum is simpler than calculating average value.

Then we made sure that the introduced changes did not decrease network accuracy. The new structure is shown in Fig. 3.

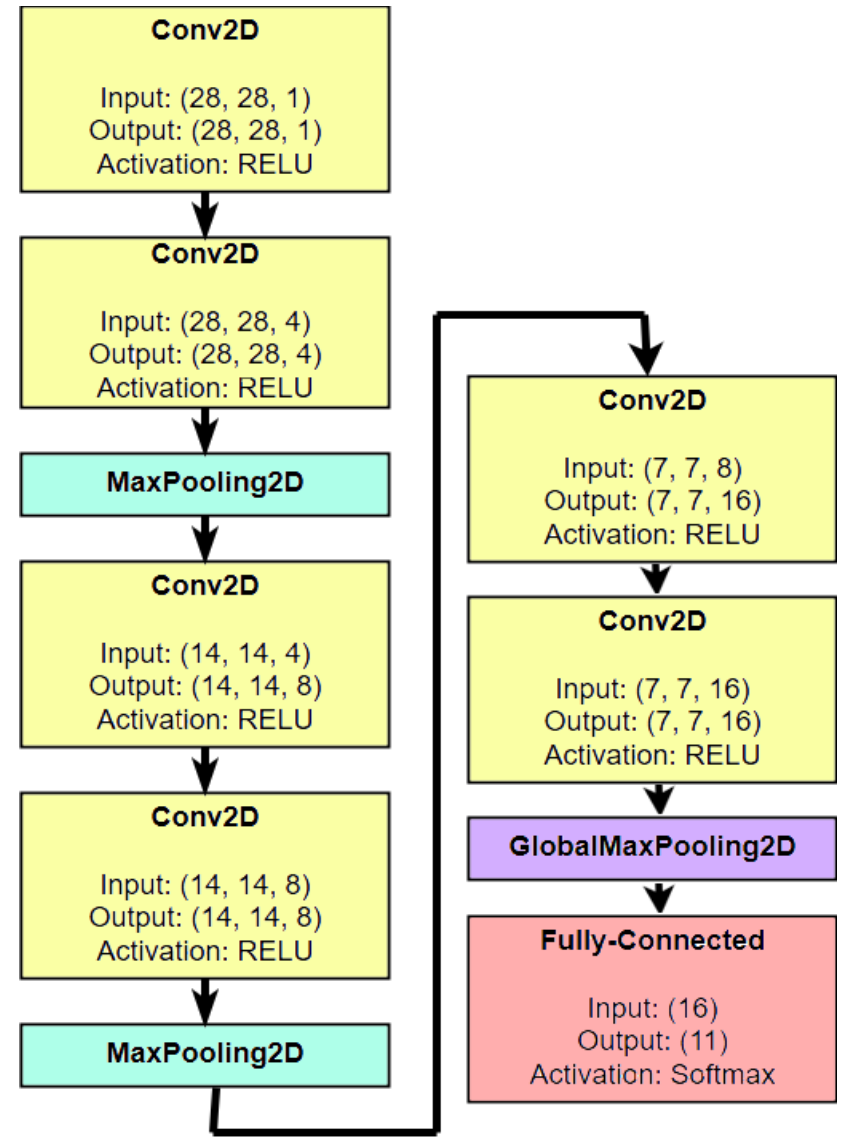

Fig. 3. Low Weights Digit Detector (LWDD) neural network structure

Neural network modification resulted in reduction of number of weights from 25 thousand to about 4.5 thousand. This allowed storing all weights in FPGA internal memory.

When using modified MNIST dataset with augmentations, neural network fairly quick trains to 96\% accuracy.

\section{CONVERSION OF FLOATING POINT CALCULATIONS TO FIXED POINT}

In neural networks, calculations are traditionally performed with floating point: either on GPU (fast) or CPU (slow), for example, using float32 type. When implemented at hardware level, floating-point calculations are slower than fixed point because of difficulty in controlling mantissa and exponent for various operations.

Consider the first layer of Convolution type neural network (Fig. 4), which is the basic block of most convolutional neural networks.

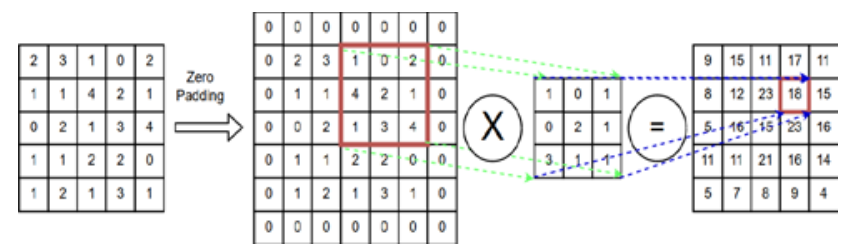

Fig. 4. Schematic presentation of Convolution operation 
Input of this layer is a two-dimensional matrix $28 \times 28$ (original image) with values from interval $[0 ; 1)$.

It is also known that if $a \in[-1 ; 1]$ и $b \in[-1 ; 1]$, then $a * b \in[-1 ; 1]$

Formula for calculating certain pixel in position $(i, j)$ of second layer, provided that Convolution dimension is $3 \times 3$, looks as follows:

$$
\begin{aligned}
& n_{i j}=b+ \\
& w_{00} * p_{i-1, j-1}+w_{01} * p_{i-1, j+0}+w_{02} * p_{i-1, j+1}+ \\
& w_{10} * p_{i+0, j-1}+w_{11} * p_{i+0, j+0}+w_{12} * p_{i+0, j+1}+ \\
& w_{20} * p_{i+1, j-1}+w_{21} * p_{i+1, j+0}+w_{22} * p_{i+1, j+1}
\end{aligned}
$$

Since weights $w_{i, j}$ and bias $b$ are known, we can calculate potential minimum $m n$ and maximum $m x$ on the second layer. Let $M=\max (|m x|,|m n|)$. If we divide $w_{i, j}$ and $b$ by value $M$, we guarantee that for any input data configuration value on the second layer does not exceed 1. Let us call this value $M$ reduction coefficient of the layer. For the second layer, we see the same situation as for the first, namely, value at layer input lies in the interval $[0 ; 1)$, so we can apply the same proof.

We can easily show that for the proposed neural network after all weight reductions on the last layer, position of maximum on the last neuron will not change; that is, neural network will operate equivalently to the neural network without reduction from the point of view of floating-point calculations.

After applying reduction to each layer, we can move from floating point calculations to fixed point calculations, as we know exactly range of values at each computation stage.

We use the following notation to represent $N$ bit numbers:

$$
x b=\left\lfloor x \cdot 2^{N}\right\rfloor
$$

If $z=x+y$,

then addition: $\quad z^{\prime}=x b+y b=\left\lfloor x \cdot 2^{N}\right\rfloor+\left\lfloor y \cdot 2^{N}\right\rfloor=$ $\left\lfloor(x+y) * 2^{N}\right\rfloor=\left\lfloor z * 2^{N}\right\rfloor=\lfloor z b\rfloor$,

multiplication: $\quad z^{\prime}=x b \cdot y b=\left\lfloor x \cdot 2^{N}\right\rfloor \cdot\left\lfloor y \cdot 2^{N}\right\rfloor=$ $\left\lfloor(x \cdot y) \cdot 2^{N} \cdot 2^{N}\right\rfloor=\left\lfloor z \cdot 2^{N} \cdot 2^{N}\right\rfloor=\left\lfloor z b \cdot 2^{N}\right\rfloor$,

that is, we divide multiplication result by $2^{N}$ to get real value, or just shift it by $\mathrm{N}$ positions.

If we sort through all possible input images aiming at the potential minimum and maximum, we can get very large reduction coefficients, and accuracy will rapidly decrease from layer to layer. As the result, big width will be needed for fixed point representation of weights and intermediate computational results. To avoid this, we can use all (or some part) of image training set as the most likely ones to find maximum and minimum values on each layer. As experiments have shown, usage of training set makes it possible to lessen reduction coefficients sufficiently. At that, we should scale up coefficients by a small margin, either focusing on value of 3 sigma or increasing maximum by several percent.
However, under certain conditions, overflow and violation of calculated range is possible. In order to handle this issue, detecting such cases and replacing overflowed values with maximum for given layer is needed when hardware is implemented. Anyway, this can be achieved by minor modification of convolution unit.

Rounding errors inevitably arise for fixed point calculations with limited width of weights and intermediate results. Such errors accumulate from layer to layer and can lead to incorrect operation of neural network. We consider classification error as incorrect work for comparison with mathematical model rather than with real result. To validate correct operation, all test images should be processed both with floating-point mathematical model and with fixed point mathematical model (or Verilog HDL benchmark); then responses at output of neural network should be compared. Ratio of number of mismatches to total number of tests is measure of error for given width of weights and intermediate results. To choose bit width, we can focus on bit width at which number of errors is 0 .

Two different strategies are possible for calculating with the use of convolution blocks:

- rounding after each elementary operation of addition and multiplication;

- calculation with full accuracy and rounding at the very end of convolution operation.

Two experiments were carried out to determine the most effective approach (see Table 1). To achieve zero difference from mathematical model, number storage requires 17 bits in case of rounding at the beginning, and only 12 bits in case of rounding at the end.

Table 1

Inaccuracy for different rounding strategies as compared to mathematical model

\begin{tabular}{|c|c|c|}
\hline $\begin{array}{l}\text { Bit width of } \\
\text { weight values }\end{array}$ & $\begin{array}{l}\text { Rounding } \\
\text { in process (\%) }\end{array}$ & $\begin{array}{l}\text { Rounding } \\
\text { at the end (\%) }\end{array}$ \\
\hline 10 & 32.03 & 1.96 \\
\hline 11 & 15.03 & 0.65 \\
\hline 12 & 11.76 & 0 \\
\hline 13 & 9.15 & 0 \\
\hline 14 & 3.27 & 0 \\
\hline 15 & 1.96 & 0 \\
\hline 16 & 0.65 & 0 \\
\hline 17 & 0 & 0 \\
\hline 18 & 0 & 0 \\
\hline
\end{tabular}

Rounding after each operation slightly increases performance, and significantly increases memory overhead. Therefore, in general, it is advantageous to perform rounding operation after convolution block.

\section{ADDITIONAL OPTIMIZATIONS OF CALCULATIONS}

To increase the performance, a number of techniques are applied that made it possible to reduce the number of cycles required for one image classification. 
1) Increasing of convolution blocks number: If there is enough free space in FPGA, we can improve the performance by increasing the number of convolution blocks, thereby multiplying productivity. Consider the second convolutional block in the proposed neural network LWDD. There are 4 of 28x28 images at the layer input, and 16 blocks of weights are given. To calculate the set of outputs for this layer, also consisting of 4 images, we have to perform four multiplications of the same set of pixels by different sets of weights. If there is only one convolutional block, this takes at least 4 cycles, but if there are 4 such blocks, then only one clock cycle is needed, thus Convolution layer calculation speeds up 4 times.

2) Shift register: To perform an elementary convolution operation, we have to get values of 9 neighboring pixels from an input image, and then next 9 pixels, 6 of which have already been received in the previous step (see Fig. 5). To shorten the time for the necessary data call up, shift register is developed to keep new data at their input and at the same time to "push out" old data. Thus, each step requires only 3 new values instead of 9 .
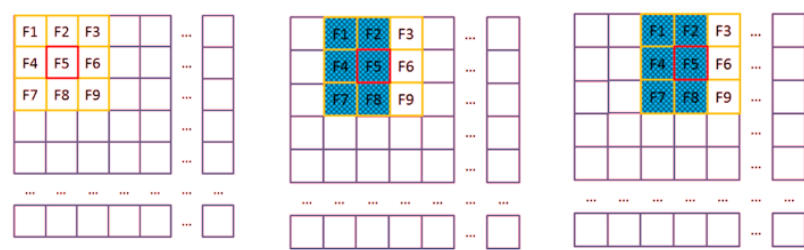

Fig. 5. Shift register operation: blue indicates data for previous convolution operation obtained at the previous step

3) Storing of all data for one Convolution operation at the same address: When we call up data that are necessary for calculations, one clock cycle is used for each value. Therefore, in order to reduce time spent on downloading required data, as well as convenience of access, prior to putting into internal memory of FPGA, data are stacked in blocks of 9 pieces, after which they are accessible at one address. With such memory arrangement, we can perform the extraction of weights in one clock cycle and, thus, speed up calculations for convolutional and fully connected layers. Example is shown in Fig. 6.

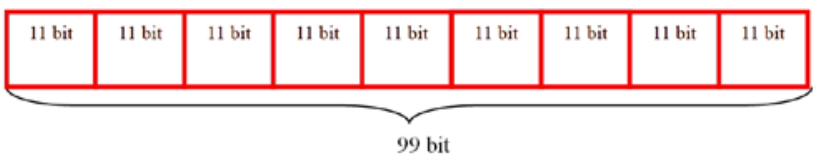

Fig. 6. Storage of all layer weights as single block

\section{EXPERIMENTAL RESULTS}

Project was successfully synthesized in Cyclone IV FPGA based on based on student debug board De0-Nano. Details on logic cells and memory usage are given in Table 2.

Images are processed in real time; original image is displayed along with the result. Classification of one image requires about 230 thousand cycles; we achieved overall processing speed with large margin of 150 frames/sec.

If performance is insufficient and spare logic cells are available, we can speed up calculations by adding convolution blocks that perform computations in parallel, as shown in [15].

Table 2

Information on resources used for device on FPGA after Place \& Route stage

\begin{tabular}{|c|c|c|c|c|}
\hline & $\begin{array}{c}\text { Logic } \\
\text { cells } \\
\text { (avail.: } \\
22320)\end{array}$ & $\begin{array}{c}\text { 9-bit } \\
\text { element } \\
\text { s (132) }\end{array}$ & $\begin{array}{c}\text { Internal } \\
\text { memor } \\
\text { y } \\
\text { (avail.: } \\
608256 \\
\text { bit) }\end{array}$ & $\begin{array}{c}\text { PLLs } \\
(4)\end{array}$ \\
\hline $\begin{array}{c}\text { Original } \\
\text { image } \\
\text { transform. }\end{array}$ & $\begin{array}{c}964 \\
(4 \%)\end{array}$ & $\begin{array}{c}0 \\
(0 \%)\end{array}$ & $\begin{array}{c}0 \\
(0 \%)\end{array}$ & $0(0 \%)$ \\
\hline $\begin{array}{c}\text { Neural } \\
\text { network }\end{array}$ & $\begin{array}{c}4014 \\
(18 \%)\end{array}$ & $\begin{array}{c}23 \\
(17 \%)\end{array}$ & $\begin{array}{c}285428 \\
(47 \%)\end{array}$ & $0(0 \%)$ \\
\hline $\begin{array}{c}\text { Weights } \\
\text { database }\end{array}$ & $\begin{array}{c}0 \\
(0 \%)\end{array}$ & $\begin{array}{c}0 \\
(0 \%)\end{array}$ & $\begin{array}{c}70980 \\
(12 \%)\end{array}$ & $0(0 \%)$ \\
\hline $\begin{array}{c}\text { Intermediate } \\
\text { storage }\end{array}$ & $\begin{array}{c}1 \\
(<1 \%)\end{array}$ & $\begin{array}{c}0 \\
(0 \%)\end{array}$ & $\begin{array}{c}214448 \\
(35 \%)\end{array}$ & $0(0 \%)$ \\
\hline Total used & $\begin{array}{c}5947 \\
(27 \%)\end{array}$ & $\begin{array}{c}23 \\
(17 \%)\end{array}$ & $\begin{array}{c}371444 \\
(61 \%)\end{array}$ & $\begin{array}{c}2 \\
(50 \%)\end{array}$ \\
\hline
\end{tabular}

Video that demonstrates the results is available on Youtube [16]. Verilog code is available on GitHub [17].

\section{SUPPORT}

This research was supported by Russian Foundation for Basic Research in the frame of project No.17-07-00409.

\section{REFERENCES}

[1] Very Deep Convolutional Networks for Large-Scale Image Recognition https://arxiv.org/pdf/1409.1556v6.pdf

[2] Delving Deep into Rectifiers: Surpassing Human-Level Performance on ImageNet Classification http://arxiv.org/abs/1502.01852

[3] Going Deeper with Convolutions http://www.cs.unc.edu/ wliu/papers/GoogLeNet.pdf

[4] Deep Residual Learning for Image Recognition http://arxiv.org/abs/1512.03385

[5] U-Net: Convolutional Networks for Biomedical Image Segmentation http://arxiv.org/abs/1505.04597

[6] Abadi M. et al. TensorFlow: A System for Large-Scale Machine Learning. OSDI. 2016. V. 16. pp. 265-283.

[7] Howard A. G. et al. Mobilenets: Efficient convolutional neural networks for mobile vision applications //arXiv preprint arXiv:1704.04861. 2017.

[8] Iandola F. N. et al. SqueezeNet: AlexNet-level accuracy with 50x fewer parameters and $<0.5 \mathrm{MB}$ model size //arXiv preprint arXiv:1602.07360. - 2016.

[9] Han S., Mao H., Dally W. J. Deep compression: Compressing deep neural networks with pruning, trained quantization and huffman coding //arXiv preprint arXiv:1510.00149. 2015. 
[10] Jouppi N. Google supercharges machine learning tasks with TPU custom chip //Google Blog, May. 2016. V. 18.

[11] http://www.terasic.com.tw/cgi-

bin/page/archive.pl?Language=English\&CategoryNo=165 $\&$ No $=593 \&$ PartNo $=2$ (access date: 15.02 .2018 )

[12] Y. LeCun, L. Bottou, Y. Bengio, and P. Haffner. Gradientbased learning applied to document recognition." Proceedings of the IEEE, 86(11):2278-2324, November 1998.

[13] Regularization of Neural Networks using DropConnect, 2013, https://cs.nyu.edu/ wanli/dropc/dropc.pdf

[14] Wilhelm Burger, Mark J. Burge (2010). Principles of Digital Image Processing Core Algorithms. Springer
Science \& Business Media. pp. 110-111. ISBN 978-184800-195-4.

[15] Solovyev R.A., Kustov A.G., Rukhlov V.S., Shelokov A.N., Puzyrkov D.V. Apparatnaya realizaciya svyortochnoj nejronnoj seti v PLIS na baze vychislenij s fiksirovannoj tochkoj (Hardware implementation of a convolutional neural network in FPGAs based on fixed point calculations). Izvestiya YUFU. Tekhnicheskie nauki. 2017. No. 7 (192). pp. 186-197.

[16] URL: https://www.youtube.com/watch?v=Lhnf596o0cc (access date: 15.02.2018)

[17] URL: https://github.com/ZFTurbo/Verilog-Generator-ofNeural-Net-Digit-Detector-for-FPGA 